\title{
Niveles de nitrógeno ureico sanguíneo y su relación con la preñez en alpacas
}

\section{Levels of blood urea nitrogen and its relationship with pregnancy in alpacas}

\author{
Edith Ancco G. ${ }^{1,4}$, Manuel Hinostroza G. ${ }^{2}$, Carlos Quispe E. ${ }^{2}$, Carlos Gómez B. ${ }^{3}$
}

\section{Resumen}

El objetivo del estudio fue determinar las diferencias entre tres intervalos de clase obtenidos a partir de los niveles de nitrógeno ureico sanguíneo (NUS) y la tasa de preñez en alpacas durante la estación reproductiva. El estudio se realizó en una cooperativa comunal de Cerro de Pasco, Perú a una altitud de 4318 msnm en febrero de 2017. Se evaluaron 52 alpacas adultas con más de dos partos. Las muestras de sangre se tomaron en el día de la monta única. Los niveles de NUS se determinaron mediante un kit comercial por colorimetría y la preñez fue determinada mediante ultrasonografía a los 35 días de la monta. Los niveles de NUS variaron entre 15.07 y $46.88 \mathrm{mg} / \mathrm{dl}$ y la tasa de preñez fue de $59.6 \%$. La tasa de preñez estuvo asociada a los niveles más bajos y más altos de nitrógeno ureico sanguíneo.

Palabras clave: alpacas; nitrógeno ureico; preñez; reproducción; mortalidad embrionaria

\section{Abstract}

The aim of this study was to determine the differences between three class intervals obtained from the levels of blood urea nitrogen (BUN) and the pregnancy rate in alpacas during the reproductive season. The study was conducted in a community cooperative in Cerro de Pasco, Peru at an altitude of $4318 \mathrm{~m}$ in February 2017. Fifty-two adult alpacas with more than two parturitions were evaluated. Blood samples were taken on the day of the single mount. BUN levels were determined using a commercial colorimetry kit and

${ }^{1}$ Programa de Doctorado en Ciencia Animal, Universidad Nacional Agraria La Molina, Lima, Perú

${ }^{2}$ Escuela Profesional de Medicina Veterinaria y Zootecnia, Universidad Peruana Los Andes, Junín, Perú

${ }^{3}$ Departamento de Nutrición, Facultad de Zootecnia, Universidad Nacional Agraria La Molina, Lima, Perú

${ }^{4}$ E-mail: edith_ag18@hotmail.com

Recibido: 11 de marzo de 2018

Aceptado para publicación: 4 de septiembre de 2018 
pregnancy was determined by ultrasonography 35 days post- mounting. The BUN levels varied between 15.07 and $46.88 \mathrm{mg} / \mathrm{dl}$ and the pregnancy rate was $59.6 \%$. The pregnancy rate was associated with lower and higher levels of blood urea nitrogen.

Key words: alpaca; urea nitrogen; pregnancy; reproduction; embryonic mortality

\section{INTRODUCCIÓN}

Una gran extensión de los pastizales de las zonas altoandinas utilizadas para la alimentación de los camélidos sudamericanos (CSA) está en franco proceso de deterioro debido al sobrepastoreo y mal manejo (FAO, 2005). Los CSA en general se alimentan de pastos de baja calidad nutritiva; sin embargo, su alta capacidad adaptativa desde el punto de vista nutricional y metabólica han hecho posible su desarrollo en ambientes que destacan por periodos prolongados de forraje de pobre disponibilidad y de baja calidad nutricional (San Martín, 1996). Estos periodos críticos coinciden con el último tercio de gestación, el parto y el inmediato empadre (Van Saun y Herdt, 2014). A pesar de estas condiciones, si bien la fecundidad es alta, las tasas de preñez y de natalidad se encuentran por debajo del $50 \%$, atribuido entre otros factores al factor nutricional (Vaughan y Tibary, 2006). Adicionalmente, se conoce que el $50 \%$ de la mortalidad embrionaria ocurre antes de los 35 días de gestación (Fernández-Baca et al., 1970).

Los pastizales ofrecen en estos periodos críticos entre 9 y $12 \%$ de proteína cruda (PC) en la dieta de los CSA (San Martín, 1996; Quispe et al., 2015), requiriéndose no menos del 14\% durante la estación reproductiva (Van Saun, 2006; Fowler, 2010). Por otro lado, la eficiencia alimenticia referida al mayor tiempo de retención del alimento en el tracto digestivo de las alpacas y a la mayor tasa de tránsito de la fase líquida en los compartimientos uno y dos (10.4\%) que en ovinos (7.7\%), favorece una mayor eficiencia del crecimiento microbiano, aseguran- do que una mínima cantidad de energía sea destinada para mantenimiento de la población microbiana (Heller et al., 1986; San Martín, 2015). La digestión de la gran cantidad de proteína microbiana que llega al intestino delgado permite que el grupo carboxilo de sus aminoácidos origine glucosa y el grupo amino genere nitrógeno ureico sanguíneo (Olazabal et al., 2015), determinando que los niveles de glucosa y nitrógeno ureico sanguíneo (NUS) en CSA sean mayores que en ovinos y mucho mayor que en vacunos.

El efecto de la nutrición sobre la reproducción se ve reflejada en vacas lecheras alimentadas con alto contenido proteico en la dieta, que eleva los niveles de NUS afectando el mantenimiento de la preñez en estadios tempranos (Butler, 1998, 2000). Este problema no se ha determinado en alpacas, que a pesar del bajo contenido proteico en la dieta, poseen concentraciones superiores a $20 \mathrm{mg} / \mathrm{dl}$ de NUS (Siguas et al., 2007), en comparación con otros rumiantes cuyos niveles varían entre 10 y $20 \mathrm{mg} / \mathrm{dl}$. La concentración de NUS no se modifica en los CSA por los niveles de proteína consumidos y no se ha detectado su efecto sobre la salud del animal (Robinson et al., 2006).

Los altos niveles de NUS en los CSA no se deberían necesariamente al alto contenido de proteína en la dieta sino a su mayor eficiencia alimenticia. Por otro lado, no se disponen de estudios que evidencien la relación entre los niveles de NUS y la preñez. El objetivo del presente estudio fue, por lo tanto, determinar diferencias entre intervalos de clase de acuerdo con los niveles de NUS y su relación con la tasa de preñez en alpacas durante la estación reproductiva. 


\section{Materiales y Métodos}

El estudio fue realizado en la Cooperativa comunal «San Pedro de Racco», ubicada en el distrito de Simón Bolivar, Cerro de Pasco, Perú, a una altitud de $4318 \mathrm{msnm}$. Se colectaron muestras de sangre de 52 alpacas hembras adultas con más de dos partos, clínicamente sanas, pertenecientes al grupo de majada de 600 animales, durante la estación reproductiva (febrero-marzo de 2017).

La colecta de sangre (3-5 $\mathrm{ml})$ se hizo de la vena yugular el día del empadre con los animales en ayunas y fueron almacenadas en cajas herméticas a $8{ }^{\circ} \mathrm{C}$ hasta su traslado al laboratorio. Las muestras fueron centrifugadas a $504 \mathrm{~g}$ durante $15 \mathrm{~min}$ para separar el suero, que fue almacenado a $-20{ }^{\circ} \mathrm{C}$ hasta su análisis. La determinación de nitrógeno ureico sanguíneo (NUS) en $\mathrm{mg} / \mathrm{dl}$ fue realizada por colorimetría, utilizando el kit comercial urea salicilato (Valtek ${ }^{\circledR}$, Chile). La preñez fue determinada por ultrasonografía transrectal a los 35 días del empadre mediante observación de la vesícula embrionaria después de una sola monta. Se utilizó un ecógrafo Tringa Linear Vet (Esaote, Holanda) con transductor transrectal lineal 7.0 MHz. Se utilizaron seis machos adultos de conocida fertilidad para el empadre, tratando que cada macho sirva un número similar de hembras.

Los datos fueron analizados mediante estadística descriptiva. Se hizo una prueba de normalidad para determinar la distribución normal de los datos de NUS. Se generaron tres intervalos de clase de acuerdo a los límites mínimos y máximos de los niveles de NUS y se evaluó su asociación con la tasa de preñez mediante una prueba de Chi cuadrado a un nivel de significancia de 0.05 . Se estableció una relación indirecta entre ambas variables basada en las diferencias entre los intervalos de clase y sus respectivas tasas de preñez. En el análisis estadístico se utilizó SAS v. 9.0 y SPSS v. 15.0 .
Resultados

La media y desviación estándar del NUS en el primer día del empadre fue de $21.65 \pm$ $5.29 \mathrm{mg} / \mathrm{dl}$ (rango: $15.07-46.88 \mathrm{mg} / \mathrm{dl}$ ). El Cuadro 1 muestra los tres intervalos de clase estimados y los promedios que corresponden a los datos de NUS en la población bajo estudio. Estos intervalos fueron la base para estimar las diferencias con la prueba de Chi cuadrado y relacionarlas con la tasa de preñez.

El $59.6 \%$ de las hembras resultó gestante a los 35 días de la monta. La mayor tasa de preñez (82.4\%) ocurrió cuando los valores del NUS eran entre 19.67 y 21.77 $\mathrm{mg} / \mathrm{dl}$ (Clase 2) y la menor cuando los niveles fueron superiores a $21.77 \mathrm{mg} / \mathrm{dl}$ (Clase 3 ) $(\mathrm{p}<0.05)$.

Cuadro 1. Intervalos de clase estimados que corresponden a los datos de nitrógeno ureico sanguíneo (NUS) en 52 alpacas postparto

\begin{tabular}{ccc}
\hline $\begin{array}{c}\text { Intervalo } \\
\text { de clase }\end{array}$ & $\begin{array}{c}\text { Rango de NUS } \\
(\mathrm{mg} / \mathrm{dl})\end{array}$ & Promedio \\
\hline 1 & $15.07-19.47$ & 17.34 \\
2 & $19.67-21.77$ & 20.72 \\
3 & $21.77-46.88$ & 26.60 \\
\hline
\end{tabular}

Cuadro 2. Relación entre los niveles de nitrógeno ureico sanguíneo y la tasa de preñez 35 días pos-monta partir de tres intervalos de clase $(\mathrm{n}=52)$

\begin{tabular}{ccc}
\hline $\begin{array}{c}\text { Intervalo } \\
\text { de clase }\end{array}$ & $\begin{array}{c}\text { Alpacas } \\
(\mathrm{n})\end{array}$ & $\begin{array}{c}\text { Preñez } \\
(\%)\end{array}$ \\
\hline 1 & 17 & $41.2^{\mathrm{c}}$ \\
2 & 17 & $82.4^{\mathrm{a}}$ \\
3 & 18 & $55.6^{\mathrm{b}}$ \\
\hline \multicolumn{4}{c}{$, \mathrm{b}, \mathrm{c}$ Letras diferentes dentro de columnas } \\
indican diferencias & entre & intervalos \\
$(\mathrm{p}<0.05)$ & &
\end{tabular}




\section{Discusión}

Niveles altos de NUS en rumiantes provocan alteraciones a nivel del tracto reproductivo, impidiendo la implantación embrionaria y provocando la pérdida embrionaria en los estadios iniciales de la gestación (Butler, 1998, 2000), lo cual podría estar ocurriendo en la alpaca. El promedio de NUS en alpacas durante el posparto de $21.65 \mathrm{mg} / \mathrm{dl}$ se encuentra dentro del rango de referencia para la especie $(9-34 \mathrm{mg} / \mathrm{dl})$, según Fowler y Zinkl (1989). Sin embargo, hubo alpacas dentro de la Clase 3 con niveles de hasta $46.88 \mathrm{mg} / \mathrm{dl}$, valores que se encuentra fuera del rango establecido. Por otro lado, Siguas et al. (2007) reportaron niveles de $42.1 \mathrm{mg} / \mathrm{dl}$ en alpacas adultas durante la estación de lluvias, la cual coincide con la estación de trabajo de este estudio, en tanto que Olazábal et al. (2015) encontraron concentraciones de $43.0 \mathrm{mg} / \mathrm{dl}$ en crías de alpacas.

El presente estudio se hizo durante la época de lluvias, donde la oferta alimenticia es mayor comparado con la época seca, de allí que se asume que el contenido proteico de la dieta es mayor y, por ende, los niveles de NUS; sin embargo, el contenido de proteína cruda en las pasturas altoandinas en esta época no excede el 12\% (San Martín, 1996; Quispe et al., 2015), porcentaje por debajo de los requerimientos proteicos de la alpaca en la estación reproductiva (Van Saun, 2006). A esto se suma que el promedio del consumo en CSA es inferior al de otros rumiantes en cerca del 30\% (San Martín, 1996). Los resultados sugieren que las alpacas metabolizan la urea de manera diferente a otros rumiantes y tienen una intrínseca tasa metabólica elevada de utilización de proteína o alguna combinación de estos factores (Van Saun, 2008).

El $59.6 \%$ de preñez a un solo servicio con machos de conocida fertilidad en estación reproductiva coincide con otros estudios
(Fernández Baca, 1991; Bravo, 2010). Las diferencias en tasa de preñez según la concentración de NUS indican que niveles altos afectarían la preñez en alpacas, tal como sucede en vacas lecheras. Por otro lado, la menor tasa de preñez registrada en alpacas con los menores valores de NUS podría estar relacionada a que estos animales no estarían cubriendo sus requerimientos y se encuentran en desbalance desde el punto de vista fisiológico, lo que puede estar afectando las funciones reproductivas (Van Saun, 2008).

\section{Conclusión}

Se observa una probable relación entre los niveles de nitrógeno ureico sanguíneo (NUS) y la tasa de preñez en alpacas adultas.

\section{Agradecimientos}

Agradecimiento especial a la Cooperativa Comunal «San Pedro de Racco» por brindar las facilidades logísticas para el desarrollo del estudio.

\section{Literatura Citada}

1. Bravo PW, Diaz D, Alarcón $V$, Ordoñez C. 2010. Effect of the reproductive state of female alpacas on embryonic mortality rate. Am J Vet Res 71: 1096-1099. doi: 10.2460/ajvr.71.9.1096

2. Butler WR. 1998. Review: effect of protein nutrition on ovarian and uterine physiology in dairy cattle. J Dairy Sci 81: 2533-2539. doi: 10.3168/jds.S00220302(98)70146-8

3. Butler WR. 2000. Nutritional interactions with reproductive performance in dairy cattle. Anim Reprod Sci 60-61: 449-457. doi: 10.1016/S03784320(00)00076-2 
4. [FAO] Organización de las Naciones Unidas para la Alimentación y la Agricultura. 2005. Situación actual de los camélidos sudamericanos en el Perú. Proyecto de Cooperación Técnica en apoyo a la crianza y aprovechamiento de los Camélidos Sudamericanos en la Región Andina. [Internet]. Disponible en: $\mathrm{http} / / /$ tarwi.lamolina.edu.pe/ emellisho/ zootecnia_archivos/situacion\%20alpcas\%20 peru.pdf

5. Fernández-Baca S, Hansel W, Novoa C. 1970. Embryonic mortality in the alpaca. Biol Reprod 3: 243-251. doi: 10.1093/biolreprod/3.2.243

6. Fernández-Baca S. 1991. Origen, evolución y status actual. En: FernándezBaca S (ed). Avances y perspectivas del conocimiento de los camélidos sudamericanos. Santiago de Chile: FAO. p 91-99.

7. Fowler M, Zinkl D. 1989. Reference ranges for hematologic and serum biochemical values in llamas (Lama glama). Am J Vet Res 50: 2049-2053.

8. Fowler M. 2010. Medicine and surgery of camelids. $3^{\text {rd }}$ ed. USA: WileyBlackwell. $644 \mathrm{p}$.

9. Heller R, Cercasov V, von Engelhardt $W$. 1986. Retention of fluid and particles in the digestive tract of the llama (Lama guanacoe f. glama). Comp Biochem Phys A 83: 687-691. doi: 10.1016/03009629(86)90710-3

10. Olazábal J, Moscoso J, Franco F, San Martín F. 2015. Relación entre glucosa y nitrógeno ureico en llamas. En: VII Congreso Mundial de Camélidos Sudamericanos. Puno, Perú.

11. Quispe C, Flores E, Naupari J. 2015. Selectividad y composición química de las dietas de alpacas y ovinos bajo pastoreo mixto. En: VII Congreso Mundial de Camélidos Sudamericanos. Puno, Perú.
12. Robinson JJ, Ashworth CJ, Rooke JA, Mitchell LM, McEvoy TG. 2006. Nutrition and fertility in ruminant livestock. Anim Feed Sci Tech 126: 259276. doi: 10.1016/j.anifee-dsci.2005.08.006

13. San Martín F. 1996. Nutrición de camélidos sudamericanos y su relación con la reproducción. Rev Argentina Prod Anim 16: 305-312.

14. San Martín F. 2015. Adaptación nutricional y metabólica de los camélidos sudamericanos. En: VII Congreso Mundial de Camélidos Sudamericanos. Puno, Perú.

15. Siguas O, Páucar R, Olazábal J, San Martín F. 2007. Valores bioquímicos sanguíneos en alpacas en dos épocas del año en condiciones de Huancavelica: aportes al perfil metabólico de la especie. En: XX Reunión de la Asociación Latinoamericana de Producción Animal (ALPA). Cusco, Perú.

16. Van Saun $R$. 2006. Nutrient requirements of South American camelids: a factorial approach. Small Ruminant Res 61: 165-186. doi: 10.1016/ j.smallrumres.2005.07.006

17. Van Saun R. 2008. Effect of nutrition on reproduction in llamas and alpacas. Theriogenology 70: 508-514. doi:10.1016/ j.theriogenology.2008.04.025

18. Van Saun R, Herdt T. 2014. Nutritional assessment. In: Llama and alpaca care. Medicine, surgery, reproduction, nutrition, and herd health. Nutritional assessment. USA: Saunders. p 100-123.

19. Vaughan JL, Tibary A. 2006. Reproduction in female South American camelids: a review and clinical observations. Small Ruminant Res 61: 259-81. doi: 10.1016/j.smallrumres.2005.07.015 